\title{
Effect of Thermal Radiation on Ignition Time and Critical Temperature of a Single Sodium Droplet
}

\author{
Felix Ilesanmi Alao, ${ }^{1}$ Kolawole Sunday Adegbie, ${ }^{1}$ \\ and Matthew Oluwafemi Lawal ${ }^{2}$ \\ ${ }^{1}$ Department of Mathematical Sciences, Federal University of Technology, P.M.B 704, \\ Akure 340252, Nigeria \\ 2 Department of Mathematics, Adeyemi College of Education, Ondo 351001, Nigeria \\ Correspondence should be addressed to Felix Ilesanmi Alao,dupsan65@yahoo.com
}

Received 29 September 2010; Revised 12 December 2010; Accepted 10 January 2011

Academic Editor: Harvinder S. Sidhu

Copyright (C) 2011 Felix Ilesanmi Alao et al. This is an open access article distributed under the Creative Commons Attribution License, which permits unrestricted use, distribution, and reproduction in any medium, provided the original work is properly cited.

The present study addresses the problem of ignition of a single sodium droplet, which is an important issue for the nuclear facilities safety. The study follows the approach of previous works and extends the results of those papers to the case of radiative heat loss. The contribution of the thermal radiation is taken into account based on the P-1 approximation for thermal radiation transfer. An extension of solutions of the existing model is obtained in the presence of radiative heat loss for ignition time and critical temperature by exploiting the sensitivity of the process to large chemical activation energy. Different qualitative effects of varying the dimensionless convective heat loss parameter with ignition time and critical temperature are presented in the graphs. The results show that the inclusion of additional heat sink mechanism, that is, radiative heat loss, causes significant delays in the ignition time and reduces the critical temperature with respect to results of previous studies.

\section{Introduction}

There are many investigations on thermal ignition in the literature. The one of utmost concern is that of ignition of sodium droplet because of its extensive use as a working fluid in the design of nuclear facilities. Although basic research on droplet combustion has been conducted, some questions that arose from these previous works suggest the need for further studies, (Grosan and Pop [1], Morewitz et al. [2]). The problem of interest focuses on the effect of thermal radiation on ignition of a single sodium droplet with initial radius $r_{s}$ and ambient temperature $T_{0}$ in an oxidizing atmosphere with $T_{\infty} \leq T_{0}$ the behaviour of the droplet radius squared is still linear with time under certain conditions. The tendency of the square of the droplet radius is reproduced by imposing the quasisteady regime (QSR) for the gas 
phase. Sodium droplet temperature $T$ is assumed spatially uniform but temporally varying because of its high thermal conductivity $\lambda$ which is characterized by low vapour pressure.

However, at low vapour pressure, the thermal inertial region close to the droplet in the gas phase is much smaller than that of the liquid phase. The spray spatial scale is specified by the boundaries of the combustion chamber, and vaporization scale is characterized by the droplet sizes; therefore, the difference in these scales is of several orders of magnitude kind of problem, but they are not the focus of this work (Fachini [3]). Many fluids involved in industrial applications have a non-Newtonian behaviour. On the other hand, if the processes take place at high temperature, radiation effects cannot be neglected (Modest [4]).

Makino [5] investigated the theory and experimental comparisons of the ignition delay and limit of ignitability of a single sodium droplet. He concluded that the ignitable range expands with increasing temperature and decreasing Nusselt number. Also, Makino and Fukada [6] studied ignition and combustion of a single sodium droplet experimentally by using a falling droplet. They discovered that the ignition delay time increases first gradually and then rapidly, with decreasing initial temperature and / or oxygen concentration and reaches the limit of ignitability. In another development, Sazhin et al. [7] considered initial heat flux between fuel droplet and a gas using a new approach to application of the shell model for simulation of diesel fuel autoignition. Their problem was rectified by replacing the gas thermal conductivity. Consequently, it was assumed that the effect of thermal radiation on heating and evaporation of semitransparent diesel fuel droplets is shown to be considerably smaller when compared with the case of block opaque.

The purpose of this study is to discover how thermal radiation affects ignition time and critical temperature of a single sodium droplet. The contribution of the thermal radiation is taken into account based on the P-1 approximation for thermal radiation transfer, Sazhin et al. [8]. The governing equation is highly nonlinear ordinary differential equation, which is nondimensionalized. Of a particular interest is the new closed-form solution obtained for ignition time in the presence of thermal radiation. Consequently, main attention is concentrated on the situation where delays might occur before final ignition. We also aim to study an impact of thermal radiation on the critical temperature.

\section{Mathematical Formulation of the Problem}

The contribution of thermal radiation is of great importance in assessing safety features of nuclear facilities and thermal ignition in a combustible fuel droplet(s). Following Makino [5], in the presence of thermal radiation, the governing equation for single sodium droplet temperature is given in the form

$$
\begin{gathered}
\frac{4}{3} \pi r_{s}^{3} \rho_{F} \mathcal{C}_{p} \frac{d T}{d t}=4 \pi r_{s}^{2} q^{0} V_{F} W_{F}\left(\frac{\rho_{g} Y_{0}}{W_{0}}\right)^{n} B_{s} e^{-E / R T}-4 \pi r_{s}^{2} h\left(T-T_{\infty}\right)-4 \pi r_{s}^{2} \sigma\left(T^{4}-T_{\infty}^{4}\right), \\
T(0)=T_{0},
\end{gathered}
$$

where $r_{s}$ is the radius of single sodium droplet, $\rho$ is the density, $c_{p}$ is the specific heat, $T$ is the droplet temperature, $T_{0}$ is the temperature in an oxidizing atmosphere with $T_{\infty}\left(\leq T_{0}\right)$ and oxygen mass fraction $Y_{0}, t$ is the time, $q^{0}$ is the heat generated per unit mass of $\mathrm{Na}, V$ is the stoichiometric coefficient, $W$ is the molecular weight, $B_{s}$ is the frequency factor of the "global" surface reaction with the activation energy $E, R$ is the universal gas constant, $h$ is 
the heat transfer coefficient, $\sigma$ is the Stefan-Boltzman constant, and the subscripts $F, g, o$, designate the fuel $(\mathrm{Na})$, ambient gas, and oxygen, respectively.

Introducing the following dimensionless variables:

$$
\varepsilon=\frac{R T_{0}}{E}, \quad \tau=t \Delta, \quad \text { where } \frac{1}{\Delta}=\frac{2 r_{s} \rho_{F} C_{p} T_{0}}{6 B_{s} q^{0} V_{F} W_{F}} \cdot\left(\frac{W_{0}}{\rho_{g} Y_{o}}\right)^{n} \cdot\left(\frac{R T_{0}}{E}\right) e^{-R T_{0} / E}
$$

with asymptotic expansion

$$
T=T_{0}\left[1+\varepsilon \theta+0\left(\varepsilon^{2}\right)\right]
$$

we obtain dimensionless sodium droplet temperature (2.1) in the form

$$
\begin{gathered}
\frac{d \theta}{d \tau}=e^{\theta}-\gamma-\delta-(\alpha+\beta) \theta \\
\theta(0)=0
\end{gathered}
$$

where

$$
\begin{aligned}
& \gamma=\frac{6 N u}{\left(2 r_{s}\right)^{2} \Delta} \frac{\lambda_{g}}{\rho_{F} c_{p}} \frac{T_{0}-T_{\infty}}{T_{0}}\left(\frac{E}{R T_{0}}\right), \\
& \delta=\frac{6 \sigma}{\left(2 r_{s}\right) \rho_{F} c_{p} \Delta} \frac{T_{0}^{4}-T_{\infty}^{4}}{T_{0}}\left(\frac{E}{R T_{0}}\right), \\
& \alpha=\frac{6 N u}{\left(2 r_{s}\right)^{2} \Delta} \frac{\lambda_{g}}{\rho_{F} C_{p}}, \\
& \beta=\frac{24 T_{0}^{3}}{\left(2 r_{s}\right)^{2} \rho_{F} C_{p} \Delta} .
\end{aligned}
$$

Here, $\gamma$ and $\alpha$ are convective heat loss parameters and the parameters $\delta$ and $\beta$ are new ones and responsible for the contribution of the thermal radiation, Nusselt number $N u=2 r_{s} h / \lambda_{g}$. It is worth noting that (2.2) without $\gamma, \delta$, and $\beta$ coincides with that of Semenov's classical thermal explosion problem, Frank-Kamenetskii [9]. Whenever $\delta$ and $\beta$ are neglected in (2.2), we obtain equation (2) in Makino [5]. 


\section{Method of Solution}

Employing the results given in Makino [5], we obtain a new closed-form solution for ignition time in (2.2) as

$$
\begin{aligned}
\tau_{\text {ig }}=\sum_{k=1}^{\infty} \frac{(\gamma+\delta)^{k-1}}{k}\{1 & +\left(\frac{\alpha+\beta}{\gamma+\delta}\right) \frac{k-1}{k}+\left(\frac{\alpha+\beta}{\gamma+\delta}\right)^{2} \frac{(k-1)(k-2)}{k^{2}} \\
& \left.+\cdots+\left(\frac{\alpha+\beta}{\gamma+\delta}\right)^{k-1} \frac{(k-1)(k-2), \ldots, 2 \cdot 1}{k^{k-1}}\right\}
\end{aligned}
$$

with $[(\gamma+\delta)+(\alpha+\beta) \theta] e^{\theta}<1$.

Consequently, when $(\alpha+\beta) \gg(\gamma+\delta)\left(T_{0}=T_{\infty}\right)$, we get

$$
\tau_{\mathrm{ig}}=1+\frac{1}{4}(\alpha+\beta)+\frac{2}{27}(\alpha+\beta)^{2}+\frac{3}{128}(\alpha+\beta)^{3}+\cdots \frac{(k-1) !}{k^{k}}(\alpha+\beta)^{k-1}+\cdots
$$

Likewise, when $(\gamma+\delta) \gg(\alpha+\beta)\left(T_{0}>T_{\infty}\right)$, we get

$$
\tau_{\mathrm{ig}}=\frac{1}{(\gamma+\delta)} \ln \left(\frac{1}{1-(\gamma+\delta)}\right)
$$

It is worth noting that in the presence of radiative heat loss, ignition limit is given as $(\alpha+$ $\beta) e^{\gamma+\delta / \alpha+\beta}=e$. Therefore, critical temperature for the single droplet is obtained as

$$
\theta_{\mathrm{cr}}=1-\frac{(\gamma+\delta)}{(\alpha+\beta)}
$$

Consequently, in the absence of radiative heat loss, the ignition limit reduces to $\alpha e^{r / \alpha}=e$ as in Makino [5]. Hence, the critical temperature is given as

$$
\theta_{\mathrm{cr}}=1-\frac{\gamma}{\alpha}
$$

\section{Result}

An extension of existing results for ignition time and critical temperature for single sodium droplet in the presence of thermal radiation is presented in (3.1)-(3.3) and (3.4), respectively. The qualitative impacts of thermal radiation in this study are ascertained in Figures 1 and 2 below. We notice in Figure 1 that existence of thermal radiation causes a notable delay in the ignition time whereas in Figure 2 significant reduction in the critical temperature of the single sodium droplet is observed. 


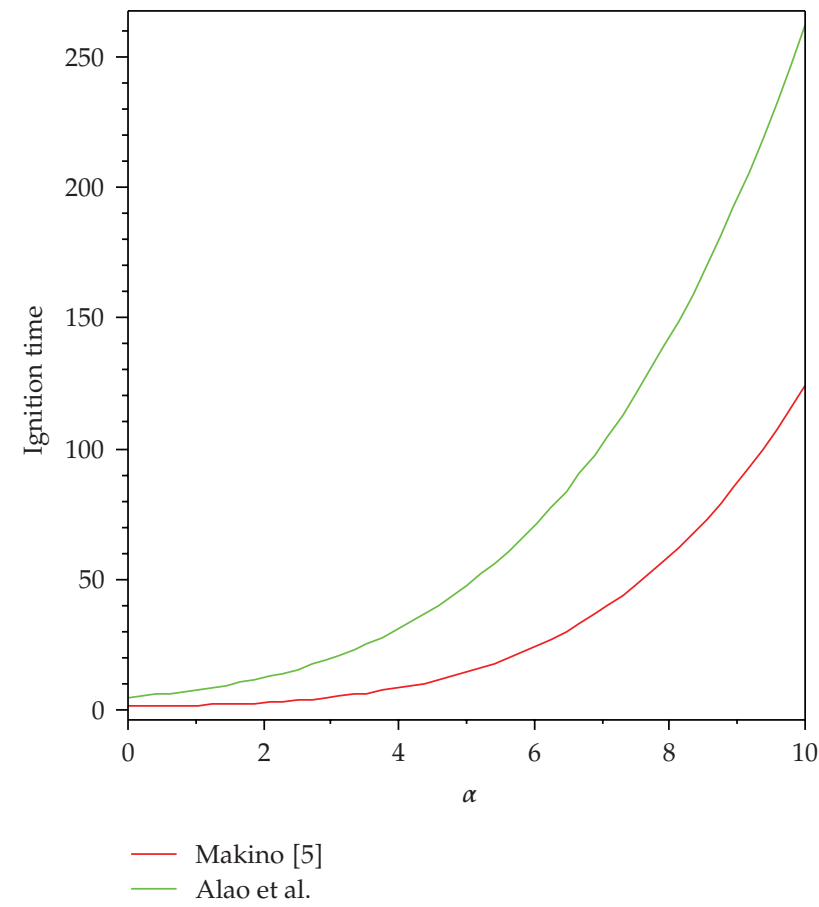

Figure 1: Comparison of ignition times: plot of (6) in Makino [5] and (3.1) in present study.

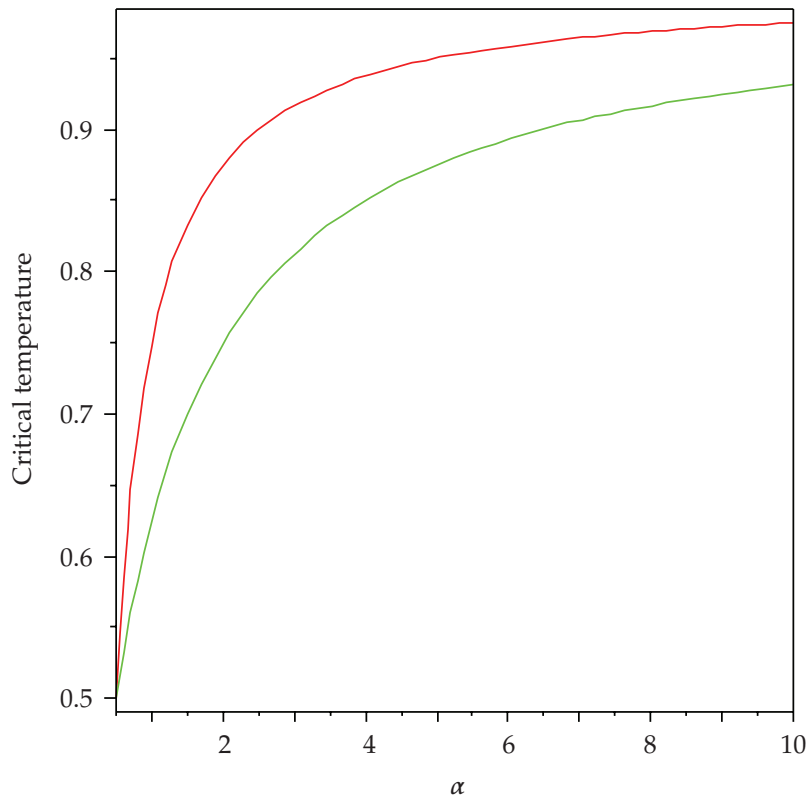

Makino [5]

- Alao et al.

Figure 2: Comparison of critical temperatures: plot of (3.4) and (3.5). 


\section{Conclusion}

In this study, the existing model is extended to include the radiative heat loss. Preliminary analyses allow us to conclude that the parameters $\delta$ and $\beta$ are responsible for the contribution of thermal radiation into the system. Explicit formulae for ignition times and critical temperature in terms of radiative heat loss parameters have been obtained. Graphical results for the ignition time and critical temperature revealed that the additional delay before ignition and reduction in critical temperature depend significantly on the inclusion of additional heat sink mechanism, that is, radiative heat loss, which is of great importance for nuclear facilities safety. Further extensions of the present approach may be pursued in the directions of further accounting for nonisobaric condition, spatial nonhomogeneities, and temperature dependence of thermophysical properties such as preexponential factor, thermal conductivity, and specific heat capacity at constant pressure.

\section{References}

[1] T. Grosan and I. Pop, "A note on the effect of radiation on free convection over a vertical flat plate embedded in a non-newtonian fluid saturated porous medium," International Journal of Applied Mechanics and Engineering, vol. 11, no. 3, pp. 715-722, 2006.

[2] H. A. Morewitz, R. P. Johnson, and C. T. Nelson, "Experiments on sodium fires and their aerosols," Nuclear Engineering and Design, vol. 42, no. 1, pp. 123-135, 1977.

[3] F. F. Fachini, "Effects of the initial droplet temperature on the vaporization process at high pressure," Journal of the Brazilian Society of Mechanical Sciences and Engineering, vol. 29, no. 1, pp. 91-98, 2007.

[4] F. Modest, Radiative Heat Transfer, Academic Press, New York, NY, USA, 2nd edition, 2003.

[5] A. Makino, "Ignition delay and limit of ignitability of a single sodium droplet: theory and experimental comparisons," Combustion and Flame, vol. 134, no. 1-2, pp. 149-152, 2003.

[6] A. Makino and H. Fukada, "Ignition and combustion of a falling, single sodium droplet," Proceedings of the Combustion Institute, vol. 30, no. 2, pp. 2047-2054, 2005.

[7] S. S. Sazhin, E. M. Sazhina, M. R. Heikal et al., "Modelling of fluid dynamics, heat transfer and combustion processes in diesel engines," in Proceedings of 18th International Conference on Science and Motor Vehicles, pp. 119-122, Yugoslav Society of Automotive Engineers, 2002.

[8] S. S. Sazhin, E. M. Sazhina, O. Faltsi-Saravelou, and P. Wild, "The p-1 model for thermal radiation transfer: advantages and limitations," Fuel, vol. 75, no. 3, pp. 289-294, 1996.

[9] D. A. Frank-Kamenetskii, Diffusion and Heat Transfer in Chemical Kinetic-Theory of Thermal Explosion, Plenum Press, New York, NY, USA, 1969. 


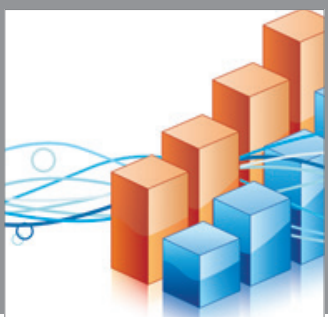

Advances in

Operations Research

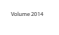

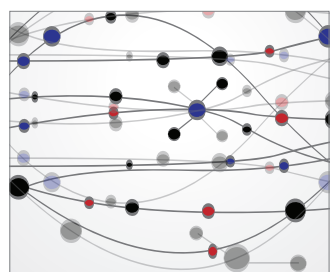

\section{The Scientific} World Journal
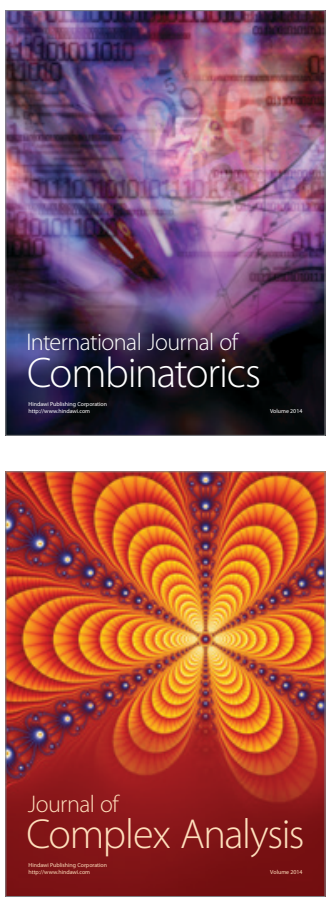

International Journal of

Mathematics and

Mathematical

Sciences
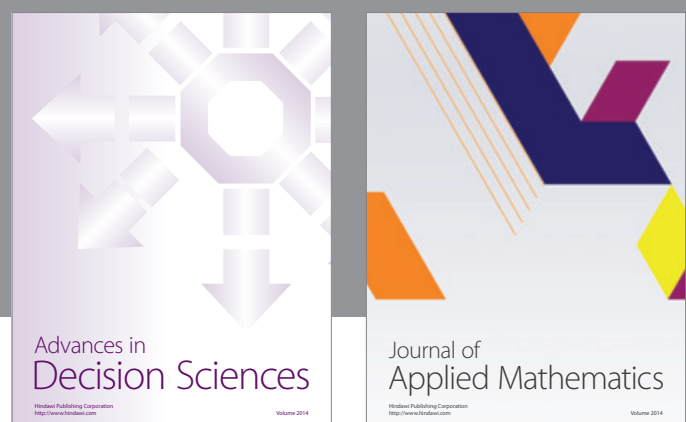

Journal of

Applied Mathematics
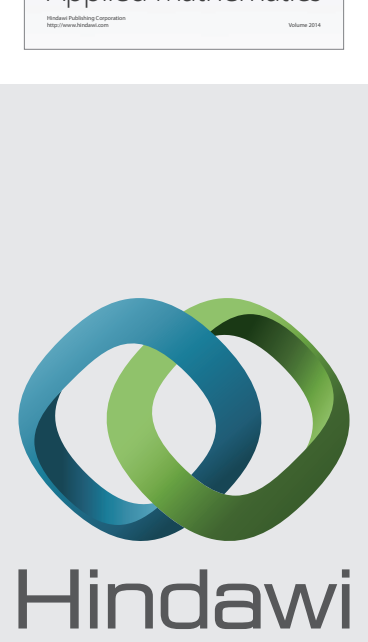

Submit your manuscripts at http://www.hindawi.com
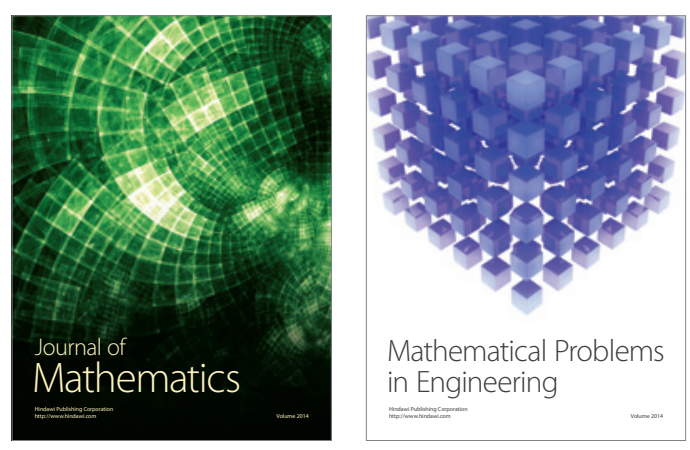

Mathematical Problems in Engineering
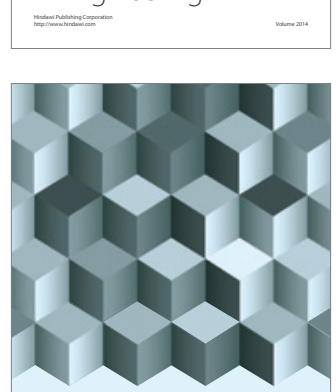

Journal of

Function Spaces
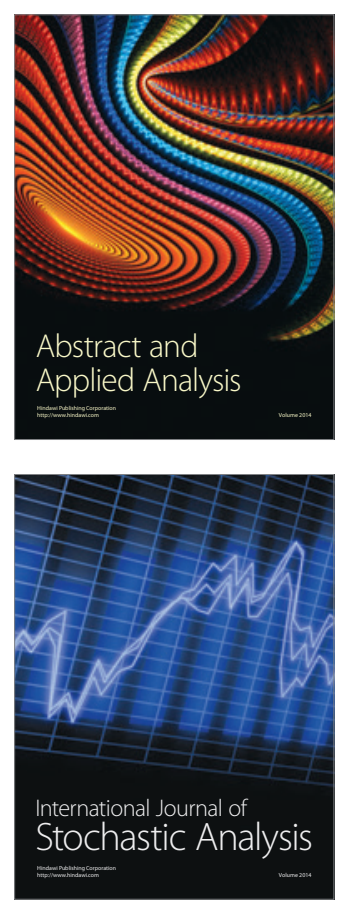

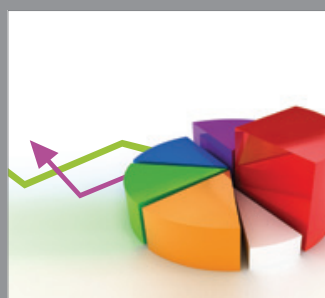

ournal of

Probability and Statistics

Promensencen
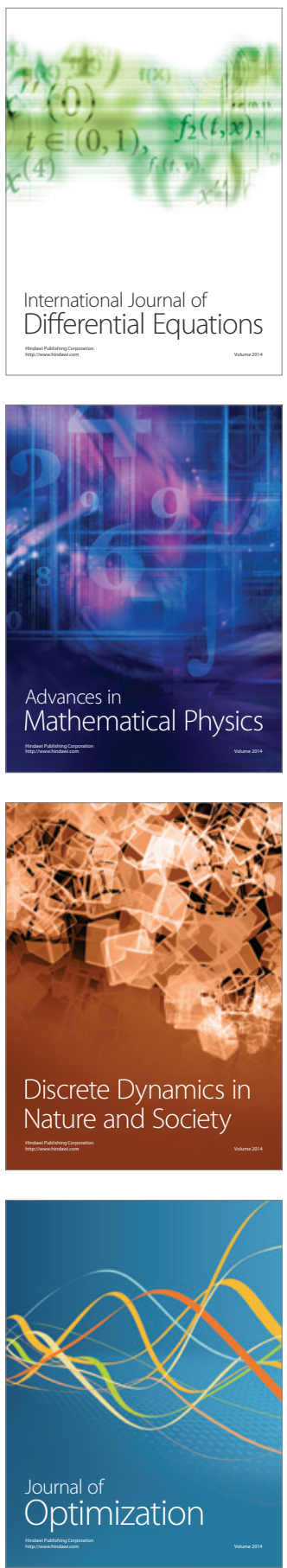